\title{
Preserved corticospinal conduction without voluntary movement after spinal cord injury
}

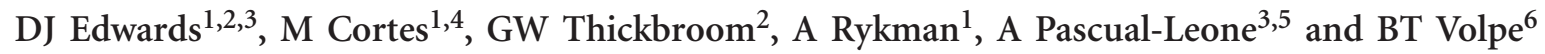

Study design: Case report.

Objectives: To identify preserved corticomotor connection in chronic spinal cord injury (SCI) in the absence of clinically observable movement.

Setting: Rehabilitation Hospital and Medical Research Institute, NY, USA.

Methods: The motor-evoked potential (MEP) response to transcranial magnetic stimulation (TMS) was recorded using surface electromyography from the right biceps brachii, extersor carpi radialis (ECR), flexor carpi radialis (FCR) and abductor pollicis brevis (APB) muscles in a 31-year-old male traumatic SCI chronic patient-ASIA B, injury level C5. Motor power scores were additionally obtained from a clinician blinded to the results of TMS.

Results: TMS could consistently elicit MEPs of normal latency, phase and amplitude, in the severely affected ECR muscle but not the similarly affected FCR muscle. The response in proximal and unaffected biceps muscle was larger than the healthy subject, whereas no response was obtained in the distal APB muscle as expected.

Conclusion: TMS can identify residual pathways not apparent from clinical assessment alone, which may have prescriptive value for rehabilitation.

Spinal Cord (2013) 51, 765-767; doi:10.1038/sc.2013.74; published online 30 July 2013

Keywords: spinal cord injury; case study; transcranial magnetic stimulation; motor evoked potential; corticospinal conduction

\section{INTRODUCTION}

Pathological studies after human spinal cord injury (SCI) have indicated that a significant number of patients retain some anatomical continuity of the spinal cord white matter across the lesion, even in the most severe cases. ${ }^{1}$ This raises the possibility that residual connectivity could be a substrate for some degree of functional recovery if it were possible to identify its presence and relevance to voluntary muscle control. We hypothesized that transcranial magnetic stimulation (TMS) might identify such latent corticomotor connections in chronic SCI. Previous studies have shown that motor-evoked potentials (MEPs) can be present in muscle with only weak activation, but they are substantially delayed and small. Here we provide evidence for a strong corticomotor connection with normal latency to a severely affected muscle (poor voluntary muscle activation) in chronic SCI.

\section{MATERIALS AND METHODS \\ Participants}

We recruited a 31-year-old (height $188 \mathrm{~cm}$ ) male with a history of traumatic SCI (diving accident) 6 years before the study (post-injury MRI, Figure 1), with otherwise negative medical history. He presented with a motor-complete sensory-discomplete (ASIA Impairment Scale grade B) C5 level of injury, and motor power (MP, Medical Research Council (MRC) scale 0-5) in the right upper limb was as follows: Elbow Flexors 5; Wrist Extensors 1; Elbow Extensors 2; Wrist Flexors 1; Finger Flexors 1; Finger Abductor 0 (these findings were confirmed by a second experienced and blinded clinician). We also studied a healthy male of similar age and height $(38$ years, $186 \mathrm{~cm}$ ) for comparison. $^{2}$ The study was approved by the Burke Rehabilitation Hospital Review Board.

\begin{abstract}
Measures
Electromyography recordings were made from the right biceps brachii (BB), extersor carpi radialis (ECR), flexor carpi radialis (FCR) and abductor pollicis brevis (APB) muscles. TMS was delivered using a MagPro device (MagVenture Inc., Atlanta, GA, USA) and figure-8-coil (MagVenture Inc., DB80) over the optimal site for each muscle, identified using a systematic search pattern of $1 \mathrm{~cm}$ steps with a pre-marked cap. The muscle response to TMS or voluntary effort was recorded using bipolar surface electrodes (SX 230, Biometrics Ltd, Ladysmith, VA, USA) with $20-400 \mathrm{~Hz}$ band-pass filter and $1000 \times$ gain on-site and digitized (Micro 1401 MkII, Cambridge Electronic Design Ltd, Cambridge, UK) at $2 \mathrm{kHz}$, then processed offline. Individual MEP waveforms were analyzed for latency and peak-to-peak amplitude using Spike 2 software (CED Version 6.02). Resting motor threshold (RMT) was determined using $2 \%$ stimulator output steps in ascending and descending order, and was defined as the intensity (\% maximal stimulator output) that elicited at least three of five evoked responses achieving $>50 \mu \mathrm{V}$ at the optimal stimulation site. MEP amplitude and latency were recorded using the average of 10 stimuli at $120 \%$ RMT for each muscle.
\end{abstract}

\footnotetext{
${ }^{1}$ Non-invasive Brain Stimulation and Human Motor Control Laboratory, Burke Medical Research Institute, Departments of Neurology and Neuroscience, Weill Medical College of Cornell University, White Plains, NY, USA; ${ }^{2}$ Australian Neuro-muscular Research Institute and Centre for Neuromuscular and Neurological Disorders, University of Western Australia, Perth, Western Australia, Australia; ${ }^{3}$ Berenson-Allen Center for Non-Invasive Brain Stimulation, Harvard Medical School, Boston, MA, USA; ${ }^{4}$ EMG and Motor Control Unit, Department of Neurology, Hospital Clinic, Universitat de Barcelona, Barcelona, Spain; ${ }^{5}$ Institut Guttmann, Universitat Autonoma de Barcelona, Barcelona, Spain and ${ }^{6}$ Feinstein Institute for Medical Research, Manhasset, NY, USA

Correspondence: Dr DJ Edwards, Non-invasive Brain Stimulation and Human Motor Control Laboratory, Burke Medical Research Institute, Departments of Neurology and Neuroscience, Weill Medical College of Cornell University, 785 Mamaroneck Avenue, White Plains, NY 10605, USA.

E-mail: dedwards@burke.org
}

Received 27 December 2012; revised 24 June 2013; accepted 27 June 2013; published online 30 July 2013 


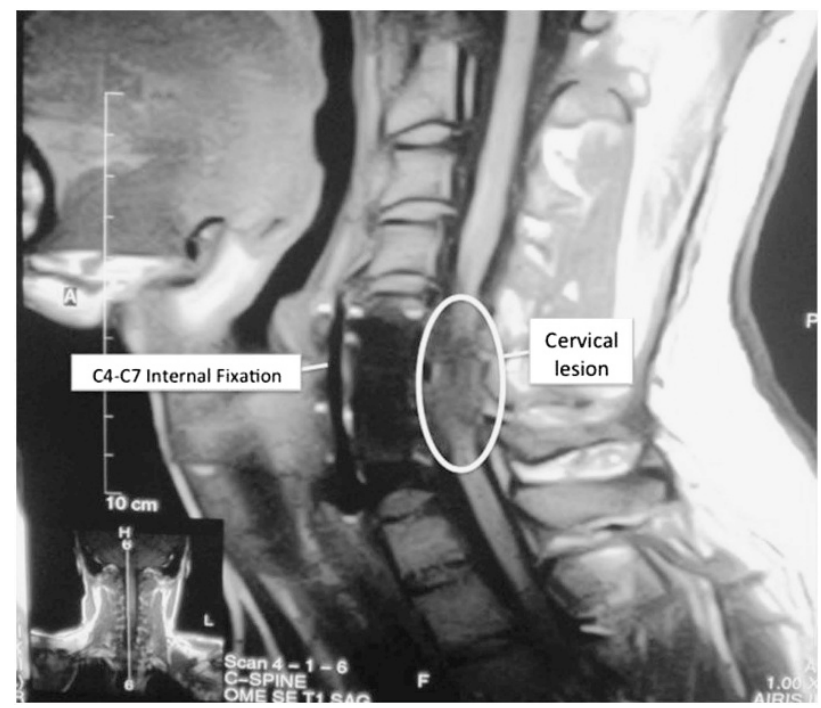

Figure $1 \mathrm{MRI}$ image of the cervical spine $\sim 18$ months post injury. Marked cervical atrophy extending from slightly superior to the midline of the C4 vertebral body, to the inferior margin at the C7-T1 level. The radiological findings are not unexpected given the time since injury, and are consistent with clinical findings.

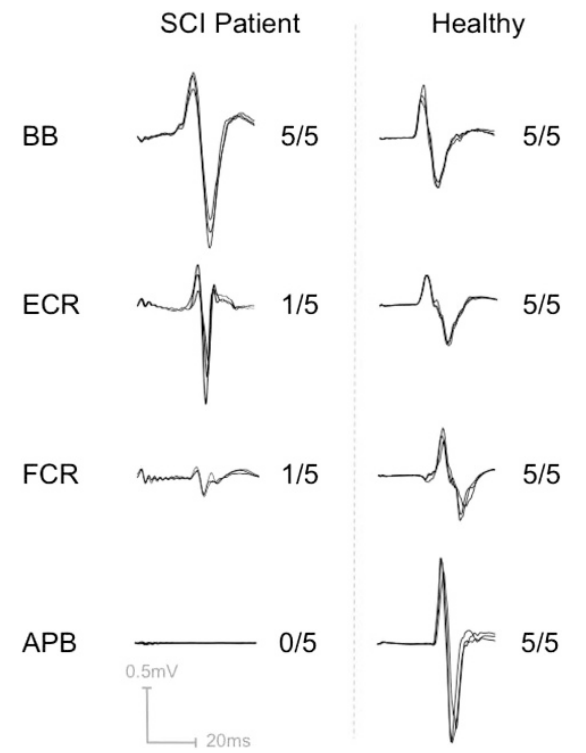

Figure 2 Sample motor-evoked potential responses to transcranial magnetic stimulation and corresponding motor power as assessed clinically (MRC scale: $0=$ no activation, $1=$ trace activation with no movement, $5=$ full muscle power) from affected and unaffected right upper extremity muscles of the in the $\mathrm{SCl}$ patient relative to a healthy age-, gender- and heightmatched subject. The distinguishing characteristics are that a muscle with only trace voluntary activation may have a strong or weak evoked response. APB, abductor pollicis brevis; $\mathrm{BB}$, biceps brachii; ECR, extensor carpi radialis; FCR, flexor carpi radialis. Note that distal muscles have typically larger responses as shown with the healthy subject, and a larger proximal response in the patient may reflect increased excitability of muscles proximal to denervated muscles.

\section{Statement of ethics}

We certify that all applicable institutional and governmental regulations concerning the ethical use of human volunteers were followed during the course of this research.

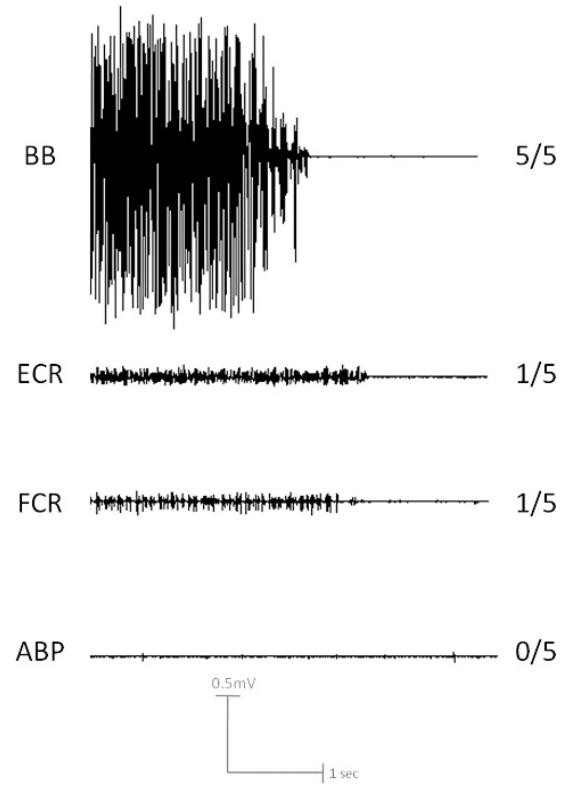

Figure 3 Electromyography (EMG) traces during attempted MVC in the chronic $\mathrm{SCl}$ patient. Note the dramatic difference in maximal voluntary EMG between the BB muscle with full power, both ECR and FCR muscles with trace activity only, and the APB muscle with no activity. EMG activity was commensurate with the clinical exam in each case $(5 / 5,1 / 5,1 / 5,0 / 5$ respectively).

\section{RESULTS}

Figure 2 shows sample-overlaid waveforms from the SCI patient at rest and the height-matched healthy male volunteer, relative to MP score (Figure 3: SCI patient EMG during maximal contraction, consistent with MP score). MEPs were present in the ECR, FCR and BB of the SCI patient $(0.61,0.07$ and $0.75 \mathrm{mV}$, respectively), and in all cases they were biphasic and of normal latency (22, 20.8 and $18.4 \mathrm{~ms})$. There was no response from the APB, even with maximal stimulator intensity. The response amplitude in the severely affected ECR was comparable to the control, and the amplitude in the biceps was greater than the control. There was an order of magnitude difference in MEP amplitude between ECR and FCR, which both had the same MP score.

\section{DISCUSSION}

We have shown in long-term stable SCI that some muscles can have normal MEPs but lack volitional contraction sufficient to generate movement, suggesting that the corticospinal projection is partially intact but cannot be engaged. Furthermore, the degree of weakness as assessed clinically does not always predict disrupted corticomotor conduction as previously thought. The apparent hyper-excitability of the biceps muscle may result from cortical reorganization involving denervated distal muscles, a phenomenon consistent with previous reports. ${ }^{3}$

It is known that MEPs may be absent even with some voluntary motor function in $\mathrm{SCI}^{4}$ but this is the first report of normal MEPs (latency, phase and amplitude) in the absence of volitional movement. The identification of residual corticomotor conduction after SCI, which can be normal even in severely affected muscles, raises the possibility that TMS could identify promising targets for rehabilitation that are not apparent from clinical assessment alone.

\section{CONFLICT OF INTEREST}

The authors declare no conflict of interest. 


\section{ACKNOWLEDGEMENTS}

The contents of the publication were developed under the NIH grants R01HD069776, R21HD060999 and K24 RR018875. We acknowledge Professor Frank Mastaglia for assistance in data interpretation and Dr Ana H Medeiros for assistance in the manuscript preparation.

Disclaimer: Dr Edwards developed the idea and was involved in each stage of experimental work and manuscript preparation. Dr Cortes and Ms Rykman contributed to data collection and manuscript preparation. Professor Pascual-Leone was involved in data interpretation and manuscript preparation. Professor Thickbroom was involved in the interpretation of the data and the drafting of the manuscript. Professor Volpe was involved with development of the idea, data interpretation and manuscript preparation.
1 Kakulas BA. A review of the neuropathology of human spinal cord injury with emphasis on special features. J Spinal Cord Med 1999; 22: 119-124.

2 Calancie B, Alexeeva N, Broton JG, Suys S, Hall A, Klose KJ. Distribution and latency of muscle responses to transcranial magnetic stimulation of motor cortex after spinal cord injury in humans. J Neurotrauma 1999; 16: 49-67.

3 Cohen LG, Bandinelli S, Topka HR, Fuhr P, Roth BJ, Hallett M. Topographic maps of human motor cortex in normal and pathological conditions: mirror movements, amputations and spinal cord injuries. Electroencephalogr Clin Neurophysiol Suppl 1991; 43: 36-50.

4 McKay WB, Stokic DS, Dimitrijevic MR. Assessment of corticospinal function in spinal cord injury using transcranial motor cortex stimulation: a review. J Neurotrauma 1997; 14: 539-548. 